\title{
AN INTRODUCTION TO THE MATHEMATICAL THEORY OF DYNAMIC MATERIALS
}




\section{Advances in Mechanics and Mathematics}

\section{VOLUME 15}

Series Editors:

David Y. Gao

Virginia Polytechnic Institute and State University, U.S.A.

Ray W. Ogden

University of Glasgow, U.K.

Advisory Editors:

I. Ekeland

University of British Columbia, Canada

S. Liao

Shanghai Jiao Tung University, P.R. China

K.R. Rajagopal

Texas A\&M University, U.S.A.

T. Ratiu

Ecole Polytechnique, Switzerland

David J. Steigmann

University of California, Berkeley, U.S.A.

W. Yang

Tsinghua University, P.R. China 


\section{AN INTRODUCTION TO THE MATHEMATICAL THEORY OF DYNAMIC MATERIALS}

By

KONSTANTIN A. LURIE

Worcester Polytechnic Institute, Worcester, MA

照 Springer 
Library of Congress Control Number: 2006940343

\author{
ISBN-10: 0-387-38278-X \\ e-ISBN-10: 0-387-38280-1 \\ ISBN-13: 978-0-387-38278-4 \\ e-ISBN-13: 978-0-387-38280-7
}

Printed on acid-free paper.

AMS Subject Classifications: 35L05, 35L70, 49S05, 49K20, 78A40, 78A48

(c) 2007 Springer Science+Business Media, LLC

All rights reserved. This work may not be translated or copied in whole or in part without the written permission of the publisher (Springer Science+Business Media, LLC, 233 Spring Street, New York, NY 10013, USA), except for brief excerpts in connection with reviews or scholarly analysis. Use in connection with any form of information storage and retrieval, electronic adaptation, computer software, or by similar or dissimilar methodology now known or hereafter developed is forbidden.

The use in this publication of trade names, trademarks, service marks, and similar terms, even if they are not identified as such, is not to be taken as an expression of opinion as to whether or not they are subject to proprietary rights.

987654321

springer.com 
To the memory of Ella 


\section{Contents}

1 A General Concept of Dynamic Materials ............ 1

1.1 The idea and definition of dynamic materials............ 1

1.2 Two types of dynamic materials .................. 2

1.3 Implementation of dynamic materials in electronics and optics 7

1.3.1 Ferroelectric and ferromagnetic materials ......... 7

1.3.2 Nonlinear optics........................ 10

1.4 Some applications of dynamic materials . ............. 11

1.5 Dynamic materials and vibrational mechanics ............ 12

References ..................................... 15

2 An Activated Elastic Bar: Effective Properties ............ 17

2.1 Longitudinal vibrations of activated elastic bar . . . . . . . . 17

2.2 The effective parameters of activated laminate $\ldots \ldots \ldots \ldots .24$

2.3 The effective parameters: homogenization............ 28

2.4 The effective parameters: the Floquet theory ............. 31

2.5 The effective parameters: discussion.................. 33

2.6 Balance of energy in longitudinal wave propagation through an activated elastic bar ........... 40

References ................................ 49

3 Dynamic Materials in Electrodynamics of Moving

Dielectrics ................................ 51

3.1 Preliminary remarks $\ldots \ldots \ldots \ldots \ldots \ldots \ldots \ldots \ldots \ldots \ldots \ldots \ldots$

3.2 The basics of electrodynamics of moving dielectrics ............................... 51

3.3 Relativistic form of Maxwell's system .............. 53

3.4 Material tensor $s$ : discussion. Two types of dynamic materials 58

3.5 An activated dielectric laminate: one-dimensional wave propagation $\ldots \ldots \ldots \ldots \ldots \ldots \ldots \ldots$ 
3.6 A spatio-temporal polycrystallic laminate: one dimensional wave propagation $\ldots \ldots \ldots \ldots \ldots \ldots \ldots \ldots$

3.7 A spatio-temporal polycrystallic laminate:

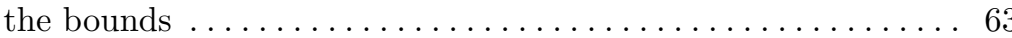

3.8 An activated dielectric laminate: negative effective material properties . . . . . . . . . . . . . . 70

3.9 An activated dielectric laminate: the energy

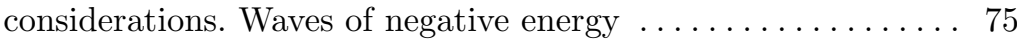

3.10 Numerical examples and discussion . . . . . . . . . . . . 81

3.11 Effective properties of activated laminates calculated via Lorentz transform. Case of spacelike interface . . . . . . . . 87

4 G-closures of a Set of Isotropic Dielectrics with Respect

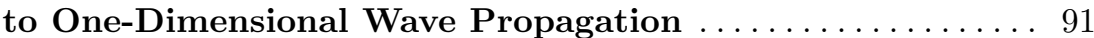

4.1 Preliminary considerations. Terminology . . . . . . . . . . . . 91

4.2 Conservation of the wave impedance through one-dimensional wave propagation. A stable $G$-closure of a single isotropic dielectric.

4.3 A stable $G$-closure of a set $U$ of two isotropic dielectrics with respect to one-dimensional

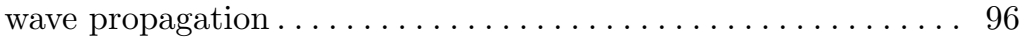

4.4 The second invariant $\mathcal{E} / M$ as an affine function; a stable $G$-closure of an arbitrary set

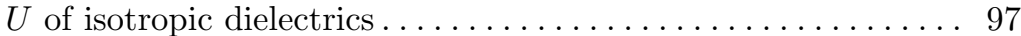

4.5 A stable $G_{m}$-closure of a set $U$ of two

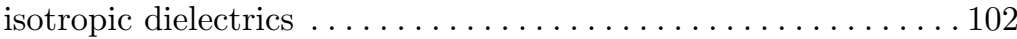

4.6 Comparison with an elliptic case $\ldots \ldots \ldots \ldots \ldots \ldots \ldots \ldots \ldots \ldots \ldots \ldots$

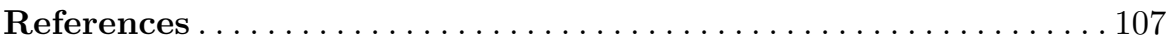

$5 \quad$ Rectangular Microstructures in Space-Time ............. 109

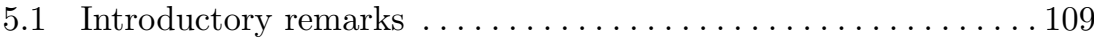

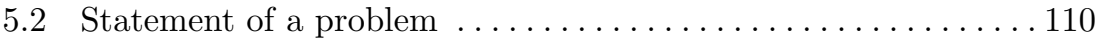

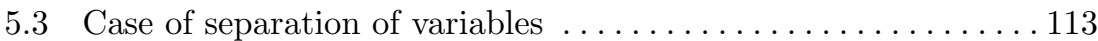

5.4 Checkerboard assemblage of materials with equal wave impedance $\ldots \ldots \ldots \ldots \ldots \ldots \ldots \ldots \ldots \ldots$

5.5 Energy transformation in the presence

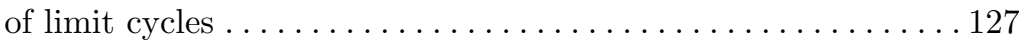

5.6 Numerical analysis of energy accumulation . . . . . . . . 131

5.7 Some remarks about discontinuous solutions

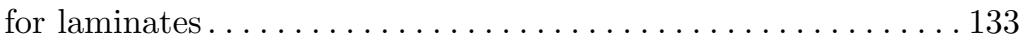


6 Some Applications of Dynamic Materials in Electrical

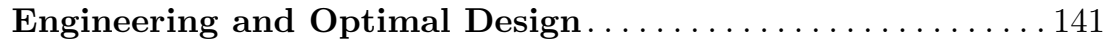

6.1 A plane electromagnetic wave propagation through an activated laminate in $3 \mathrm{D} \ldots \ldots \ldots \ldots \ldots \ldots \ldots 14 \ldots \ldots \ldots \ldots$

6.2 The homogenized equations. Elimination of the cutoff frequency in a plane waveguide . . . . . . . . . . . . . . 142

6.3 The effective material tensor and homogenized

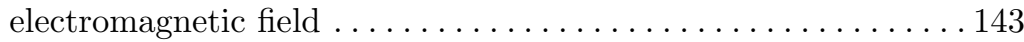

6.4 The transport of effective energy $\ldots \ldots \ldots \ldots \ldots \ldots \ldots \ldots \ldots$

6.5 On the necessary conditions of optimality in a typical hyperbolic control problem with controls in the coefficients . . 146

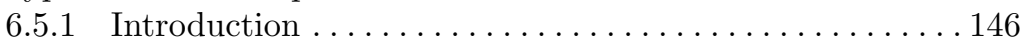

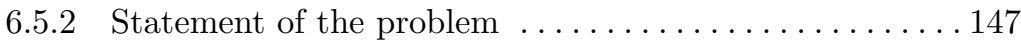

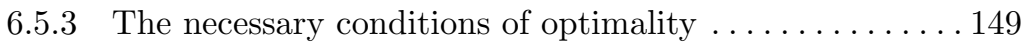

6.6 Transformation of the expression for $\Delta I$ : the strip test . . . . 153

6.7 A polycrystal in space-time . . . . . . . . . . . . . 155

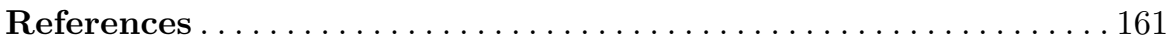

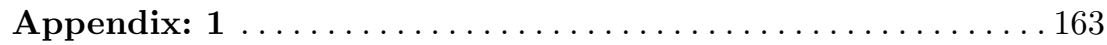

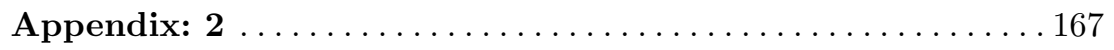

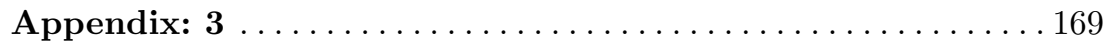

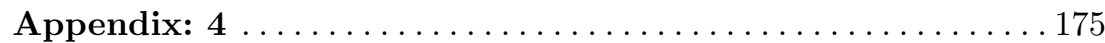

Index . . . . . . . . . . . . . . . . . . . . . . . . . . . . 179 


\section{List of Figures}

1.1 A discrete version of a transmission line. . . . . . . . . . . 3

1.2 A moving $(L C)$-property pattern - an activated composite. . . . 4

1.3 An immovable material pattern with moving original substances - a kinetic composite. ............... 4

1.4 Material laminate in space-time. ................ 6

1.5 E-P hysteresis curve for ferroelectric materials. . . . . . . . . . . 8

$1.6 \quad \mathrm{H}-\mathrm{M}$ hysteresis curve for ferromagnetic materials . . . . . . . . . 8

1.7 Single cell of ferroelectric/ferromagnetic material. . . . . . . . . . 10

1.8 Multiple cells of ferroelectric/ferromagnetic material. . . . . . . 10

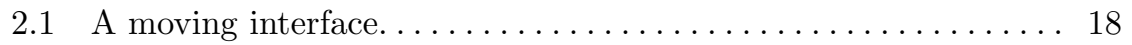

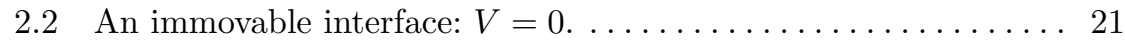

2.3 A moving interface: $|V|<a_{1} \ldots \ldots \ldots \ldots \ldots \ldots \ldots \ldots \ldots \ldots 21$

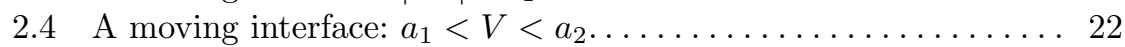

2.5 A moving interface: $-a_{2}<V<-a_{1} \ldots \ldots \ldots \ldots \ldots \ldots \ldots \ldots \ldots$

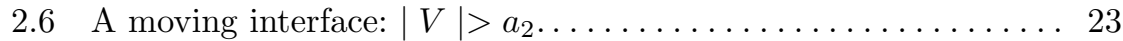

2.7 A matrix microstructure in space-time violating ineqs. (2.5) . . 23

2.8 Effective parameters $K$ versus $P$ with variable $V$ (case

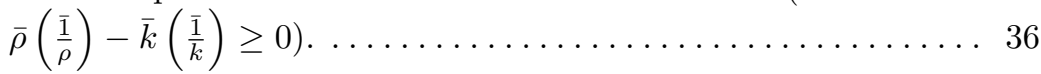

2.9 Effective parameters $K$ versus $P$ with variable $V$ (case

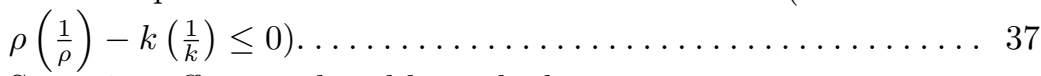

2.10 Screening effect produced by a shadow zone. . . . . . . . . 38

3.1 The "caterpillar" construction. .................... 62

3.2 The hyperbola $\mathcal{E} / M=\epsilon / \mu \ldots \ldots \ldots \ldots \ldots \ldots \ldots \ldots$

3.3 The hyperbolic strip $\epsilon_{2} / \mu_{2}>\mathcal{E} / M>\epsilon_{1} / \mu_{1}, \mathcal{E}, M \geq 0 \ldots \ldots 69$

3.4 The hyperbolic strip $\epsilon_{2} / \mu_{2}>\mathcal{E} / M>\epsilon_{1} / \mu_{1}, \mathcal{E}, M \leq 0 \ldots \ldots 70$

3.5 Effective permittivities and permeabilities of dielectric laminate with $\left(\epsilon_{1}, \mu_{1}\right)=(1,1),\left(\epsilon_{2}, \mu_{2}\right)=(9,0.1), m_{1}=0.5$, for

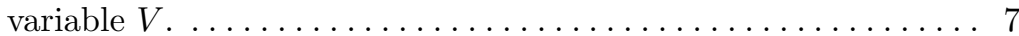


3.6 Effective wave impedance versus $V$.

3.7 Effective energy densities of dielectric laminate with $\left(\epsilon_{1}, \mu_{1}\right)=(1,1),\left(\epsilon_{2}, \mu_{2}\right)=(9,0.1), m_{1}=0.5$, for variable $V$.

3.8 Wave propagation through a fast range laminate where $V=1.3$ yields a homogenized material with negative effective coefficients.

3.9 Wave propagation through a fast range laminate where $V=4.0$ yields a homogenized material with positive effective

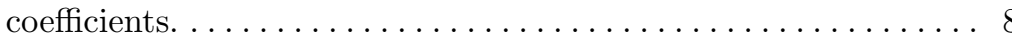

3.10 Energy densities of composites vs. $m_{1}$, for $V=1.3$. Solid line is energy density of pure material 1 , dashed line is energy

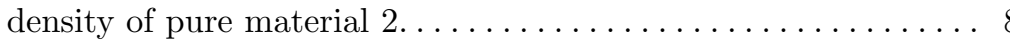

3.11 Energy densities of composites vs. $m_{1}$, for $V=4.0$. Solid line is energy density of pure material 1 ; dashed line is energy

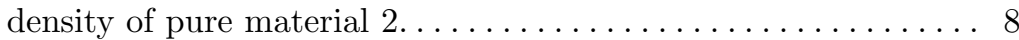

4.1 A stable $G$-closure of a set of two isotropic dielectrics of the same sign. . . . . . . . . . . . . . . . . . . . . . . 97

4.2 Case $a_{1}^{(1)}<a_{2}^{(1)}<V<a_{1}^{(2)}<a_{2}^{(2)} . \ldots \ldots \ldots \ldots \ldots \ldots \ldots 100$

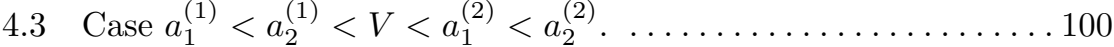

4.4 Case $a_{1}^{(1)}<a_{2}^{(1)}<V<a_{1}^{(2)}<a_{2}^{(2)}<V \ldots \ldots \ldots \ldots \ldots \ldots \ldots 101$

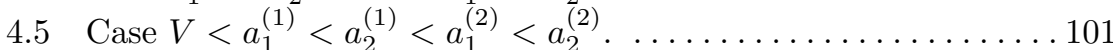

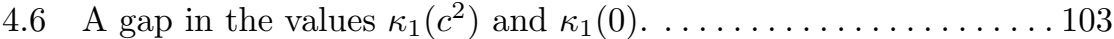

$4.7 G$-closure of a binary set of two anisotropic heat conductors in a plane. . . . . . . . . . . . . . . . . . . . . . . 104

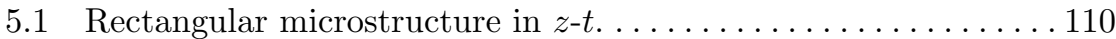

5.2 Limit cycles in the checkerboard structure with $a_{(1)}=0.6, a_{(2)}=1.1, m_{1}=0.4, n_{1}=0.5$.

5.3 Evolution of a disturbance through a structure with $m_{1}=0.4, n_{1}=0.5, a_{(1)}=0.6$, and $a_{(2)}=1.1 . \ldots \ldots \ldots \ldots 118$

5.4 Solution at time 10 of a disturbance with wide support through a structure with $m_{1}=0.4, n_{1}=0.5, a_{(1)}=0.6, a_{(2)}=1.1$, and

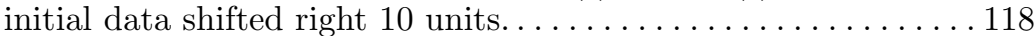

5.5 Low frequency pattern in trajectories through structure with $m_{1}=0.4, n_{1}=0.8, a_{(1)}=0.6$, and $a_{(2)}=1.1 . \ldots \ldots \ldots 120$

5.6 Closer view of wave trajectories through structure with $m_{1}=0.4, n_{1}=0.8, a_{(1)}=0.6$, and $a_{(2)}=1.1 . \ldots \ldots \ldots \ldots 120$

5.7 Structure with $m_{1}=0.4, n_{1}=0.1, a_{(1)}=0.6$, and $a_{(2)}=1.1 \ldots 121$

5.8 Wave speed as a function of $m_{1}$ and $n_{1}$ for $a_{(1)}=0.6$ and

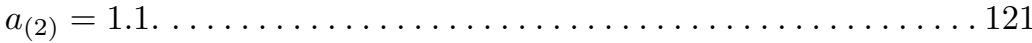

5.9 Trajectories in material with $a_{(1)}=0.6, a_{(2)}=1.1, m_{1}=0.4$

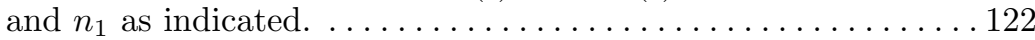

5.10 Speed versus $n_{1}$ in material with $a_{(1)}=0.6, a_{(2)}=1.1, m_{1}=0.4 .123$ 
5.11 Wave speed as a function of $a_{(2)}$ and $n_{1}$, for $a_{(1)}$ between 0.6

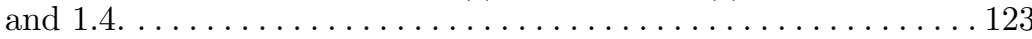

5.12 Wave speed as a function of $a_{(2)}$ and $n_{1}$, for $a_{(1)}$ between 1.5

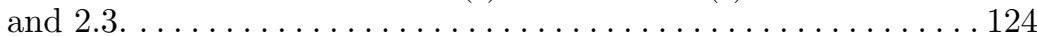

5.13 Limit cycles have speeds that are rational multiples of $\delta / \tau=1$. Here, $a_{(1)}=0.6, a_{(2)}=0.9, m_{1}=0.15 \ldots \ldots \ldots \ldots \ldots \ldots \ldots \ldots$

5.14 Wave speed $=3 / 4$ when $a_{(1)}=0.6, a_{(2)}=0.9, m_{1}=0.15$, and

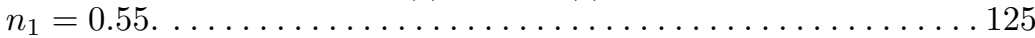

5.15 Wave speed $=2 / 3$ when $a_{(1)}=0.6, a_{(2)}=0.9, m_{1}=0.15$, and

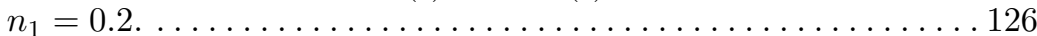

5.16 Solution at time 30 of a disturbance with wide support through a structure with $a_{(1)}=0.6, a_{(2)}=0.9, m_{1}=0.15, n_{1}=0.2$,

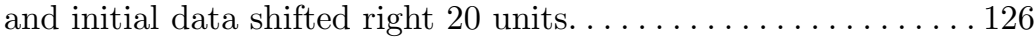

5.17 Wave speed $=1 / 2$. Use $m_{1}=0.0579, n_{1}=0.3529, a_{(1)}=$ $0.8132, a_{(2)}=0.0099$ (randomly generated parameters) . . . . 127

5.18 Wave speed $=2 / 7$. Use $m_{1}=0.8757, n_{1}=0.7373, a_{(1)}=$ $0.4096, a_{(2)}=0.0353$ (randomly generated parameters) . . . . 127

5.19 Wave speed $=2 / 5$. Use $m_{1}=0.5651, n_{1}=0.9692, a_{(1)}=$ $0.1187, a_{(2)}=4.3511$ (randomly generated parameters) . . . . 128

5.20 The bunch of characteristics in the vicinity of a limit cycle.

The spatial and temporal periods of the microstructure are taken equal to $\epsilon$, other parameters specified as $a_{(1)}=0.6, a_{(2)}=1.1, m_{1}=0.4, n_{1}=0.5 \ldots \ldots \ldots \ldots \ldots \ldots \ldots$

5.21 Characteristic paths through checkerboard material (5.36). . . 132

5.22 Solution at time 4 to $a_{(1)}=0.55, a_{(2)}=2 a_{1}, m_{1}=0.5, n_{1}=0.5 .132$

5.23 Energy variation up to time 4 for $a_{(1)}=0.55, a_{(2)}=$ $2 a_{(1)}, m_{1}=0.5, n_{1}=0.5 \ldots \ldots \ldots \ldots \ldots \ldots \ldots \ldots \ldots \ldots \ldots \ldots \ldots \ldots$

5.24 Characteristic paths through checkerboard material (5.37). . . . 134

5.25 Right going characteristic paths through material (5.37) . . . . 135

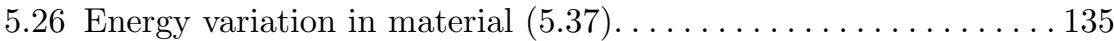

5.27 Solution at time 10 to material $(5.37) \ldots \ldots \ldots \ldots \ldots \ldots \ldots \ldots \ldots$

5.28 The pattern of characteristics in a laminate violating ineqs. (2.5)136

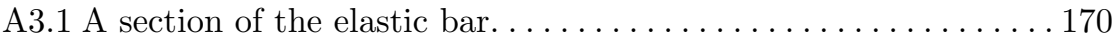

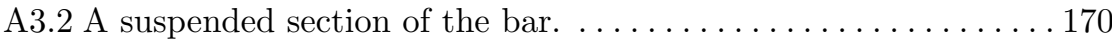

A3.3 An elastic bar as an assembly of sections. . . . . . . . . . . 171

A3.4 Rolls from two adjacent sections mounted on the common axis. 172

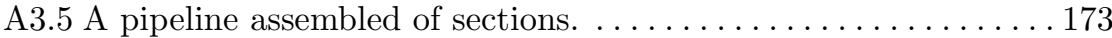




\section{Preface}

This book has emerged from the study of a new concept in material science that has been realized about a decade ago. Before that, I had been working for more than 20 years on conventional composites assembled in space and therefore adjusted to optimal material design in statics. The reason for that adjustment is that such composites appeared to become necessary participants in almost any optimal material design related to a state of equilibrium.

A theoretical study of conventional composites has been very extensive over a long period of time. It received stimulation through many engineering applications, and some of the results have become a part of modern industrial technology. But again, the ordinary composites are all about statics, or, at the utmost, are related to control over the free vibration modes, a situation conceptually close to a static equilibrium.

The world of dynamics appears to be quite different in this aspect. When it comes to motion, the immovable material formations distributed in space alone become insufficient as the elements of design because they are incapable of getting fully adjusted to the temporal variation in the environment. To be able to adequately handle dynamics, especially the wave motion, the material medium must itself be time dependent, i.e. its material properties should vary in space and time alike. Any substance demonstrating such variation has been termed a dynamic material [1].

The wave propagation through dynamic materials may be accompanied by a number of special effects that are unthinkable with regard to purely static materials mentioned above. In general, dynamic materials may be thought of as assemblages of conventional materials distributed in space and time; particularly, they may be involved in their own material motion. When such assemblage is furnished with a microstructure, we may call it a dynamic composite, or a spatio-temporal composite, contrary to its conventional (i.e. static) counterpart.

Unlike conventional composites, dynamic materials are rarely found in mother nature: all of them known so far have come into the scene as the products of modern technology. The only exception, though of extreme signif- 
icance, is a living tissue. There is one fundamental feature that brings the two substances together: they both participate in a permanent exchange of energy and momentum with the environment and therefore appear to be thermodynamically open systems. Due to this exchange, the dynamic materials represent a suitable environment for dynamics, especially for the wave propagation. This particular feature adds much to the resources available to a designer because it makes it possible to establish an effective control over both spatial and temporal behavior of a dynamic system.

Regardless of a material implementation, it is now the time to investigate some general features of dynamic materials mathematically. A general scheme for such investigation may be similar to the one successfully tested with regard to ordinary static composites. One of the most exciting problems that received solution in this connection is the problem of material mixing in space. A study of this problem has put forth a special concept of a $G$-closure $(G U)$ of the original set $U$ of materials [2],[3],[4]. A $G$-closure is defined as a set of the effective properties of all mixtures that are produced when the original materials become intermingled on a microscale, regardless of a structural geometry. Clearly, $U \in G U$. The $G$-closures were found explicitly for a number of sets $U$ with regard to some important elliptic differential operators arising in electrostatics and in the theory of elasticity. Analytically, all of the $G$-closures known so far have been found with the aid of a special technique named the translation method [3]; it has been worked out specifically for this purpose. The knowledge of a $G$-closure is sufficient for a correct formulation of many design problems that remain ill-posed without such knowledge.

The idea of a $G$-closure has emerged from the desire to make a set of available materials complete, simply by adding all possible mixtures to it. This idea surely persists in a hyperbolic context, too, and it has been an intriguing task to investigate $G$-closures produced by some typical hyperbolic operators governing the non-stationary phenomena developing in dynamic materials. Some results of such studies are included into this book. The analysis is related to a simple wave operator

$$
\left(\rho u_{t}\right)_{t}-\left(k u_{z}\right)_{z},
$$

with coefficients $\rho, k$ being both $t$ and $z$-dependent. The problem is therefore two dimensional, with one spatial coordinate $z$ and time $t$. The operator (1) serves as a good model, similar to that given by the operator

$$
\operatorname{div} \mathcal{D} \operatorname{grad} u, \quad \mathcal{D}=\mathcal{D}(x, y),
$$

in a relevant elliptic situation. Many features of hyperbolic $G$-closures revealed through the study of (1) are quite special and substantially different from the properties of the $G$-closures associated with (2). These differences are likely to be even more pronounced in the case of higher spatial dimensions. The latter has not been investigated in detail in this introductory text; however, the very notion of a dynamic material, as well as the procedure of material 
mixing in space-time received a clear mathematical implementation in many spatial dimensions as well. Remarkably there is a fundamental physical theory, namely Maxwell's theory for moving dielectrics, that perfectly embodies dynamic materials as a natural dielectric medium capable of conducting electromagnetic waves. It was rewarding to find such a theory, so to speak, on the surface, because it immediately offered a natural classification of dynamic materials produced by two conceptually different ways of mixing in space-time. The reader will find a brief account of these ideas on the opening pages of the main text.

I fully realize that the presentation below is a first step towards an extensive theory that should unveil in the future. My purpose was to try to get a clear vision of the base ideas, and I believe that an interested reader will be able to share the excitement that I experienced while working on this beautiful subject.

\section{Acknowledgments}

This text would probably not appear without invaluable support that I had from many people. My thanks go to my dear colleague and friend, Dr. Suzanne L. Weekes, whose contribution to this new field is quite solid; many of her results are included into the text as its indefeasible part. Dr. Brian King of the Department of Electrical and Computer Engineering in Worcester Polytechnic Institute has contributed most of the text of sections 1.3, 1.4 of Chapter 1. Colleen Lewis and Elizabeth Teixeira of the Department of Mathematical Sciences in Worcester Polytechnic Institute did a magnificent job on typing and graphics. Major inspiration has come from discussions that I had with my old friend Professor Ilya I. Blekhman at all stages of this work. I am deeply obliged to my colleagues and friends for their remarkable effort.

My special thanks and admiration go to my wife Sonia, to my son Dmitri and daughter Aleksandra whose exceptional perseverance gave me inspiration and force to go through a difficult time when this text was written.

Support for the study of dynamic materials given through NSF Grants DMS-9803476, DMS-0204673, and DMS-0350240 is gratefully acknowledged.

Worcester, Massachusetts

The Author

December 2005 
XVIII Preface

\section{References}

1. Blekhman, I.I., and Lurie, K.A.: On dynamic materials. Proceedings of the Russian Academy of Sciences (Doklady) 37, 182-185 (2000)

2. Lurie, K.A.: Applied Optimal Control of Distributed Systems, Plenum Press, 499 pp (1993)

3. Lurie, K.A., and Cherkaev, A.V.: The effective characteristics of composite materials and optimal design of constructions. Advances in Mechanics (Poland), vol. 9, 3-81 (in Russian) (1986)

4. Cherkaev, A.V.: Variational Methods for Structural Optimization, Springer Verlag, xii +627 pp (2000) 


\section{A General Concept of Dynamic Materials}

\subsection{The idea and definition of dynamic materials}

The idea of composites is one of the key ideas in material science. When different substances are used as primary elements through the constructing of material assemblages, these new formations may demonstrate properties that are alien to original constituents. Of such properties, the structural anisotropy is probably the most critical. This property is created artificially, through making composites, thanks to their special microgeometry; an anisotropic composite may thus be built from isotropic original constituents. Anisotropy is vitally important for optimal design: every such design is a custom-tailored formation built purposefully to fit in the environment peculiar to a concrete working situation. Examples illustrating this are numerous; they may be found in many texts, (see, e.g., [1],[2],[3]).

Until recently, the concept of composites has been viewed as essentially static: a composite that is ordinary in a conventional sense, is assembled once and for all in space, and this assemblage remains invariable in time. This concept fits well into the problems related to a static equilibrium; however, it fails to be adequate with regard to a dynamic environment.

To work effectively in a dynamic world, a material medium should be responsive to dynamic disturbances allowing for the energy and momentum exchange take place between various parts of the system on a suitable spatiotemporal scale. It should be able to maintain selective interaction between the material property patterns and dynamic disturbances, i.e. such interaction should occur wherever and whenever necessary. This fundamental requirement could be met if we resort to a special material arrangement termed a dynamic material.

Dynamic materials are defined as formations assembled from ordinary materials distributed in space and time. When such formation is allotted with a microstructure, a dynamic material becomes a dynamic (spatio-temporal) composite. The appearance of time is special: it serves as an additional fast 
variable. The presence of such a variable combined with the fast variable spatial coordinate, transforms an ordinary composite assembled in space alone, into a dynamic composite distributed in space and time.

The dynamic disturbances whose spatio-temporal scale is much greater than the corresponding scale of the assemblage, may perceive this one as a new material allotted its own effective properties. By changing the material parameters of original substances, as well as the microgeometry, we shall be able to selectively control the dynamic processes by creating effects that are unattainable so far as we operate with ordinary materials or composites.

One may set a difference between various types of dynamic materials, and we will introduce their formal classification in Chapter 3. At the same time, such materials share one special feature that is common to all of them: they universally appear to be substantially non-equilibrium formations. To create a dynamic material, we have to maintain the energy exchange between it and its surroundings. Energy should either be pumped into the medium, or it should be extracted from it. The effective properties of dynamic materials are therefore specifically affected by the relevant energy flows. For this reason, dynamic materials themselves appear to be thermodynamically open systems; only a combination of such material and the environment may be considered as closed.

To some extent, dynamic materials fall outside a stock notion of a material as of something that can be taken into your hands, stored, moved, manufactured once for all, individualized by placing some "indelible" labels, etc. There is no such thing as "a piece of dynamic material". Instead, they would rather be "brought into the scene" and exist with the environment. For example, a TV screen on which a movie is demonstrated represents a dynamic material: our eye perceives it, through a movie performance, as a plane with the reflection properties variable in space and time. A human mechanism of vision implements a spatio-temporal averaging of a pattern of rapidly alternating sequences and thereby detects a "slow motion" carrying information stored in the movie.

The concept of dynamic materials appears to be a special realization of the idea of smart materials, i.e. substances able to respond to environmental variations by changing their properties, structure or composition, or their function both in space and time.

\subsection{Two types of dynamic materials}

Dynamic materials have originally been introduced in [4], [5] in both mechanical and electromagnetic contexts. They have been classified into two major categories termed activated and kinetic materials. The difference between such categories is fundamental, and it is best illuminated by examples. 


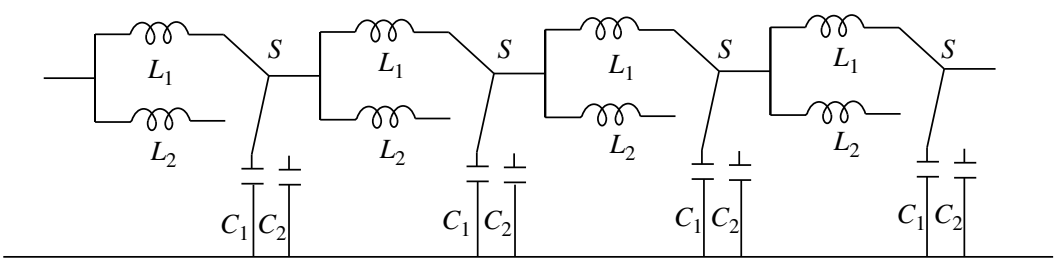

Fig. 1.1. A discrete version of a transmission line. 


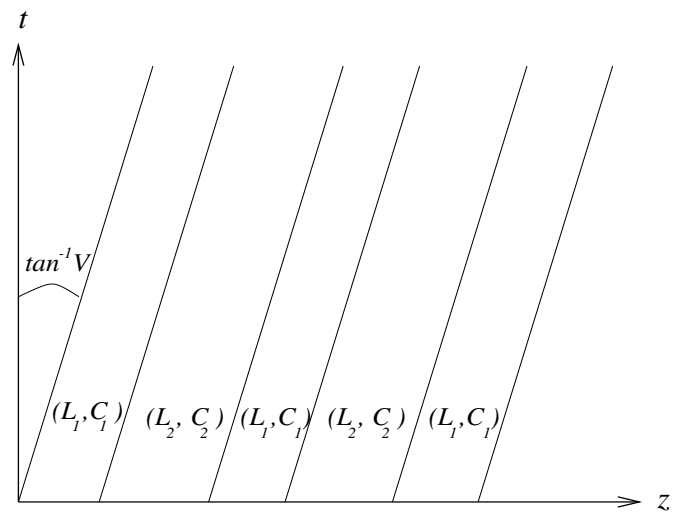

Fig. 1.2. A moving $(L C)$-property pattern - an activated composite.

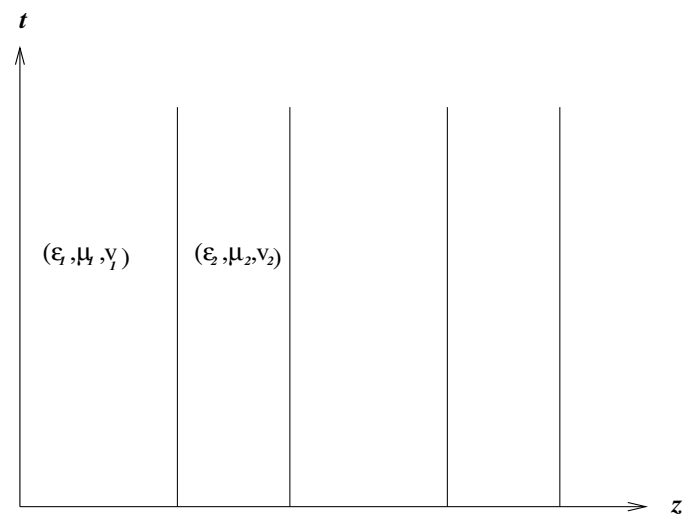

Fig. 1.3. An immovable material pattern with moving original substances - a kinetic composite.

Consider a transmission line. Its discrete version may be interpreted as an array of $L C$-cells connected in series (Fig. 1.1). Assume that each cell offers two possibilities: $\left(L_{1}, C_{1}\right)$ and $\left(L_{2}, C_{2}\right)$, turned on/off by a toggle switch $S$. If the cells are densely distributed along the line, then, by due switching, the linear inductance $L$ and capacitance $C$ of the line may become, with any desired accuracy, almost arbitrary functions of a spatial coordinate $z$ along the line, and time $t$. In particular, we may produce in a $(z, t)$-plane a periodic $L C$-laminate assembled from segments with properties $\left(L_{1}, C_{1}\right)$ and $\left(L_{2}, C_{2}\right)$, respectively (Fig. 1.2). In this figure, a periodic pattern of such segments is shown moving along the $z$-axis at velocity $V$, and this motion creates a laminated structure in space-time. It is essential that this construction does not include any motion of the material itself; what is allowed to move, is the property pattern alone. This is a pure case of activation, and activated 